\title{
The influence of counselling on patient return following uncomplicated posterior vitreous detachment
}

\begin{abstract}
Purpose To investigate the effect of patient counselling together with the use of information leaflets on the number of return visits for patients with acute, symptomatic, uncomplicated posterior vitreous detachment (PVD).

Methods The study group comprised the first 50 patients diagnosed with uncomplicated PVD in 1998 when a standard protocol of structured patient counselling and leaflet distribution was employed following diagnosis of PVD. The control group comprised the first 50 patients in 1997 diagnosed with uncomplicated PVD before structured patient counselling was in place. A retrospective analysis of casualty case notes was made comparing the two groups. The number of eye casualty attendances within 1 year of first presentation with PVD was compared in the two groups.

Results Seven patients from the control group returned because of photopsia or floaters; 3 of the 7 returned with no change in their original symptoms. Six patients from the study group returned. All had a definite change in their symptoms of photopsia or floaters. No patient in the study group who returned had old or persistent symptoms. Statistical analysis comparing return visits of patients with no change in symptoms in the two groups by Fisher's Exact Test gave a $\boldsymbol{p}$ value of 0.13 . Conclusion Patients counselled following uncomplicated PVD did not return to eye casualty in the absence of new symptoms. Patient counselling is an important part of the management of PVD because it makes patients aware of which symptoms are important predictors of serious vitreoretinal pathology.
\end{abstract}

\section{A.J. Singh \\ Department of Ophthalmology Leicester Royal Infirmary Leicester, UK}

\section{R.R. Seemongal-Dass}

Department of

Ophthalmology

Royal Halifax Infirmary

Halifax, UK

\section{A.J. Singh,}

Manchester Royal Eye Hospital

Oxford Road

Manchester M13 9WH, UK

Presented as a poster at the Royal College of Ophthalmologists Annual Congress, Harrogate, May 2000

Received: 29 March 2000 Accepted in revised form: 15 August 2000
Key words Counselling, Effective, Posterior vitreous detachment
The Kent County Ophthalmic and Aural Hospital, Maidstone provides a 24 hour, 365 day walk-in eye casualty service. Patient attendances to eye casualty are 15000 to 20000 per year. Patients presenting with symptoms of floaters and flashing lights are often found to have vitreoretinal pathology. ${ }^{1-6}$ Although up to $95 \%$ of these cases are benign, some may harbour significant pathology such as retinal breaks or detachments. ${ }^{1-6}$ Investigation of these patients is often time-consuming and utilises a significant amount of available resources. ${ }^{7}$

For the first 9 weeks in 1997 there were 2125 new patient attendances, 50 of which were diagnosed with posterior vitreous detachment (PVD). In the first 10 weeks of 1998 there were 2260 new patient attendances, 50 of which were diagnosed with PVD.

This study was designed to find out whether patient counselling affected return visits to eye casualty following a diagnosis of acute, uncomplicated PVD, and to determine whether there was a decrease in unnecessary returns in counselled patients.

\section{Methods}

From October 1997, following a diagnosis of uncomplicated PVD, patients received structured counselling by a nurse practitioner. PVD in this study was defined to be a PVD not associated with secondary complications such as retinal break, detachment or vitreous haemorrhage. A patient information leaflet, explaining with simple diagrams the relationship between PVD and symptoms of floaters and flashing lights, was given to each patient. It was emphasised that after a period of adaptation these symptoms would become less noticeable. The period of time devoted to counselling varied with each individual patient and rarely exceeded $10 \mathrm{~min}$. For most patients 5-10 min was sufficient. Moreover, a list of symptoms highly suggestive of retinal break/ 
detachment was supplied. These were: (i) an area of vision missing; (ii) a curtain-like effect in the field of vision; (iii) an increase in floaters and flashing lights. Patients who developed any of these symptoms were advised to ring the department urgently for further advice.

The study group comprised the first 50 patients in 1998 diagnosed with uncomplicated PVD when the standard protocol of patient counselling by an ophthalmic nurse practitioner and leaflet dissemination was employed. The control group comprised the first 50 patients seen in 1997 diagnosed with uncomplicated PVD before there was structured patient counselling.

A retrospective analysis of eye casualty notes was conducted comparing the number of return visits in each group. The results for a period of 1 year from first presentation with uncomplicated PVD in both groups were recorded. For those patients who did return to eye casualty, the reasons for this, as documented in their notes, were studied. Return visits for other eye problems or symptoms were not recorded as return visits for this study.

Where identified, patients with high risk factors predictive of serious vitreoretinal pathology were noted. Those at high risk are patients with: (a) high myopia, (b) lattice degeneration, (c) aphakia, (d) family history of retinal detachment and (e) hereditary vitreoretinal degenerations. ${ }^{7}$

\section{Results}

No patients re-presented with retinal breaks, retinal detachments or vitreous haemorrhage. Seven control patients and 6 study patients returned within 1 year (Table 1). All returnees in the study group did so because of new symptoms and not persistent old symptoms, whereas 3 returnees in the control group had no new symptoms (Table 2).

In each group, there were 18 male (36\%) and 32 female $(64 \%)$ patients. The mean age of patients in the study group was 64 years (range 34-85 years) and in the control group was 62 years (range $44-81$ years). In the control group 1 patient was found to have central retinal vein occlusion during follow-up. In the study group 1 patient was later diagosed with optic atrophy secondary to optic nerve sheath meningioma.

Table 1. Number of return visits for symptoms of floaters, flashing lights and loss of vision or field of vision

\begin{tabular}{lcc}
\hline & \multicolumn{2}{c}{ No. of patients } \\
\cline { 2 - 3 } No. of return visits & Control & Study \\
\hline All patients & 7 & 6 \\
One visit & 6 & 5 \\
Two visits & 1 & 0 \\
Three visits & 0 & 1 \\
Total & 7 & 6 \\
\hline
\end{tabular}

Table 2. Reasons for return to eye casualty following diagnosis of uncomplicated posterior vitreous detachment

\begin{tabular}{lcc}
\hline & \multicolumn{2}{c}{ No. of patients } \\
\cline { 2 - 3 } Reasons for return & Control group & Study group \\
\hline Increase in floaters & 3 & 4 \\
Increase in flashing lights & 0 & 1 \\
Increase in flashes and floaters & 0 & 1 \\
Decrease in visual acuity & 1 & 0 \\
Curtain effect in visual field & 0 & 0 \\
Unsure about symptoms & 3 & 0 \\
Total & 7 & 6 \\
\hline
\end{tabular}

\section{Statistical analysis}

Statistical comparison of the number of return visits for patients with no symptom change in each group $(0 / 50$ study group vs $3 / 50$ control group) was performed using Fisher's Exact Test. The one-tailed $p$ value was 0.13 . This was not highly significant.

\section{Discussion}

The mean age at presentation in both groups was in the seventh decade. This is consistent with previous studies which showed that the greatest prevalence of acute PVD was in the age group 60-69 years. ${ }^{2,5,8}$ There was a preponderance of females in this study, again in keeping with previous reports. ${ }^{2,5,8}$

Patients with acute, uncomplicated PVD without high risk factors have an extremely low incidence of secondary retinal breaks. ${ }^{1,2,8}$ Once the acute event of the PVD has passed the symptoms of floaters and flashing lights are transient and resolve with time so long as there are no major secondary complications. ${ }^{6}$ If the initial examination of a patient with PVD is normal, there is almost no risk of finding retinal breaks, holes and/or detachment during follow-up. ${ }^{1,2}$

The effect of counselling could have increased the rate of patient return in the study group but this was not the case. Greater patient awareness meant that patients were able to identify any change in the nature of their symptoms and present for prompt re-examination only when necessary. Awareness of the symptoms that are important predictors of serious vitreoretinal pathology is especially important because of the implications for early diagnosis and treatment of these conditions. ${ }^{3-8}$

All patients who returned from the study group did so because of a definite change in symptoms. None of them returned because they were unsure of what to expect in the time following the acute PVD. This, however, occurred in the control group prior to routine patient counselling $(0 / 50$ in the study group versus $3 / 50$ in the control group) (Table 2). This shows a trend, with the number of unnecessary returns in the study group being definitely decreased. We feel that this is the result of greater understanding and symptom awareness by the patients because of structured counselling and the information leaflets. 
Although the number of return visits for all patients in the two groups was similar, it is mandatory that all patients with PVD and changing symptoms be examined urgently since new pathology can occur at any time. ${ }^{4,9}$ The major worry is development of a secondary retinal break following PVD, which can present at highly variable times. ${ }^{6,8}$ In this study no new retinal breaks or detachments were detected.

The practice of structured patient counselling and the issuing of the leaflet following a diagnosis of acute PVD still occurs in the unit. Counselling is effective through self-education in reassuring anxious patients who may not fully understand the nature of their symptoms. ${ }^{10}$ The nurse practitioner while performing triage and historytaking often has the best opportunity to establish a relationship of trust with the patient and, indeed, most patients find it less intimidating to speak to nurses rather than doctors. ${ }^{11}$ Counselling seems to be an appropriate extension of the role of the specialist nurse practitioner in eye casualty. It takes only a 5-10 min discussion with each patient with PVD to reduce unnecessary visits.

In this study unnecessary visits were reduced by 3 in 2260 new patient attendances (133 per 100000$)$. In this unit, this would result in a total reduction of unnecessary visits by $20-27$ per year. Given the nature and time required to examine these patients this represents a significant saving in resources. We have not found this to be an unmanageable increase in the responsibilities of the specialist nurse practitioners; in fact they have welcomed the opportunity to have a more proactive role in patient care in eye casualty.

Counselling is important not only in decreasing unnecessary visits to eye casualty, but also in making patients aware of what symptoms are particularly important predictors of serious vitreoretinal pathology. This is especially important in this age of increased emphasis on primary prevention.
We would like to thank Siobhan Callanan and Allison Gamble for their contribution to this article, Dr J. Thompson for statistical analysis and Mr P. Richardson for his general advice.

\section{References}

1. Richardson PS, Benson MT, Kirkby GR. The posterior vitreous detachment clinic: do new retinal breaks develop in the six weeks following an isolated symptomatic posterior vitreous detachment? Eye 1999;13:373-40.

2. Diamond JP. When are simple flashes and floaters ocular emergencies? Eye 1992;6:102-4.

3. Byer NE. Natural history of posterior vitreous detachment with early management as the premier line of defense against retinal detachment. Ophthalmology 1994;101:1503-14.

4. Dayan MR, Jayamanne DG, Andrews RM, Griffiths PG. Flashes and floaters as predictors of vitreoretinal pathology: is follow-up necessary for posterior vitreous detachment? Eye 1996;10:456-8.

5. Kakerashi A, Inoda S, Shimizu Y, Makino S, Shimizu H. Predictive value of floaters in the diagnosis of posterior vitreous detachment. Am J Ophthalmol 1998;125:113-5.

6. Sharma S, Walker R, Brown GC, Cruess AF. The importance of qualitative vitreous examination in patients with an acute posterior vitreous detachment. Arch Ophthalmol 1999;117:343-6.

7. Novak MA, Welch RB. Complications of acute symptomatic posterior vitreous detachment. Am J Ophthalmol 1984;97:308-14.

8. Hikichi T, Trempe CL. Relationship between floaters, light flashes, or both, and complications of posterior vitreous detachment. Am J Ophthalmol 1994;117:593-8.

9. Kanski JJ. Complications of acute posterior vitreous detachment. Am J Ophthalmol 1975;80:44-6.

10. Pringle M, Laverty J. A counsellor in every practice. BMJ 1993;306:2-3.

11. McFarland N. Primary health care. Counselling: a reassuring practice. Nurs Stand 1990;4:47-8. 